\section{THE ACCURACY OF OPSONIC ESTIMATIONS.}

BY L. NOON, B.C. OANTAB., F.R.C.S. ENG., JOHN LUCAS WALKER STUDENT IN THE UNIVERSTTY OF CAMBRIDGE; AND

ALEXANDER FLEMING, L.R.C.P. LOND., M.R.O.S. ENG.,

ASSISTANT IN THE INOCULATION DEPARTMENT AT ST. MARY'S Hospital.

(From the Inoculation Department, St. Mary's Hospital.)

Dr. A. Reyn and Dr. R. Kjer-Petersen, in The LANCET of March 28th, publish figures on which they question whether the tuberculo-opsonic index of tuberculous patients really does present any specific variation from the normal. This is a blow aimed at the very base of all opsonic work, and we feel that an answer to the attack is not out of place.

Some sources of error to which the technique is liable are discussed by one of us in a paper which is due to appear in the Practitioner for May of this year; so that we can here confine ourselves to the criticism of Dr. Kjer-Petersen's figures, against which we produce some figures obtained at St. Mary's Hospital in the routine work of the Inoculation Department.

Our argument is arranged under four heads, which deal respectively with (1) Dr. Kjer-Petersen's position; (2) our reasons for regarding the variations of normal sera as small; (3) our reasons for regarding the variations of tuberculous sera as large; and (4) some general criticisms of Dr. Kjer-Petersen's methods.

1. Dr. Kjer-Petersen's position.-Dr. Kjer-Petersen presents us with the mean error or standard deviation of his three series of observations and proceeds to show that each series approximates to the distribution known as the "normal curve of error." This implies three propositions. (a) The calculated standard deviation of each series is a measure of the variation of the observations. (b) Nearly 70 per cent. of observations will fall within this limit, and deviations larger than four times the standard deviation are only to be expected six times in 100,000 trials. ${ }^{1}$ (c) When we are presented with the standard deviation of a series of observations, we are face to face with a number which represents deviations due to many causes. If, by any means, we can find out the standard deviation due to one of these canses acting alone, we can eliminate this (by squaring both numbers, subtracting, and taking the square root of the remainder), and so obtain the standard deviation due to the remaining causes.

From what precedes we see that Dr. Kjer-Petersen's figures are open to analysis, if we can obtain the necessary data from which to work. Before we undertake such a task we wish to draw a distinction between certain terms which we shall use. When we speak of the standard deviation presented by a series of observations, it is to be understood that the individual observed deviations may be due to errors introduced by imperfect technique, or, on the other hand, to actual variations in the biological quantity measured. Therefore we speak of a standard deviation as compounded of a mean error of observation and a mean variation of the quantity measured.

Having made these definitions we may now approach $\mathrm{Dr}$ Kjer-Petersen's figures and make use of the statistical method in which he trusts. We shall see what conclusions may be drawn from these figures.

Dr. Kjer-Petersen finds a standard deviation (from unity) in his observations on normal sera of $0 \cdot 19$, as opposed to 0.25 and 0.29 in two series of tuberculous cases. The standard deviation 0.19 , for normals, represents variations in the normal sera used and errors in the technique of opsonic estimation. One or other of these factors must be considerable. If the variations of normal sera have been more important than the errors of technique, then the difference between 0.19 and 0.25 , or 0.29 (the difference, that is,

1 It must be remarked, however, that the frailty of human nature may introduce into any collection of measurements occasional large errors which are neglected by the "law of error" in its simplest form. If such errors are rare, statisticians generally reject them frem their calculations and content themselves with recording their occurrence. For this purpose they use Chauvenet's eriterion, which they apply in order to define a limiting deviation. An observation which falls outside this limit is not included in the determination of the constants of a eurve. between normal and tuberculons sernm, is not of great value, and Dr. Kjer-Petersen's criticism is justified. If, on the other hand, the errors have been more important than the variations, then no definite conclusions can be drawn from his figures.

We propose to show that the variations of normal sera are small in comparison with the figures given above. Consequently Dr. Kjer-Petersen's work lacks accuracy. Hence we may set aside his conclnsions in favour of others which are supported by better figures.

2. The variations of normal sera are small.-The records of routine work in the Inoculation Department of St. Mary's Hospital have supplied us with 763 pairs of observations on normal sera. The samples of serum have been obtained from 60 presumably normal individuals, who from time to time offered themselves for this purpose. The difference between the counts for the two normals we have expressed as a percentage of the mean of the two counts. Bearing in mind Mr. M. Greenwood's letter, in THE LANCET of April 11th, we are careful to premise that we have attempted, not a complete mathematical treatment of these data, but an approximation, which is still near enough to justify our rejection of Dr. Kjer-Petersen's results. Our figures are distributed very nearly acccording to the normal curve of error and give a standard deviation of 10 per cent. Table I. gives the observed distribution, compared with the theoretical, which is enclosed in brackets.

\section{TABLE I.}

Within 5 per cent. $326=427$ per mille $(383)$

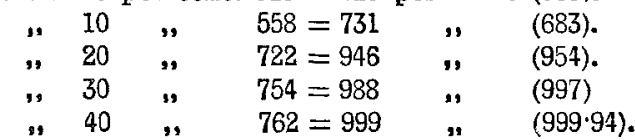

What does this standard deviation of 10 per cent. mean? It is not the standard deviation of single observations from a hypothetical mean normal; it represents two such deviations, because it measures the difference between two observations, both of which may vary. Then the value 10 per cent. is compounded of two equal standard deviations from the mean normal. Observations of the indices of normal sera are consequently subject to a standard deviation of $\sqrt{\frac{10}{2}}$, or 7 per cent. Estimations of unity, depending on the mean of two such observations, are subject to a mean error of $\sqrt{2}$, or 5 per cent.

Now this standard deviation of 7 per cent. is compounded of the mean variation of the opsonic indices of normal sera, and the mean error of observation at St. Mary's Hospital. Either of these unknown quantities must therefore be less than 7 per cent. That such a deviation is relatively small may be seen by correcting Dr. Kjer-Petersen's figures in the light of our own. Suppose for example we assume a mean variation for normals of 7 per cent. (the largest our figures admit); this corresponds to 0.07 of unity. Eliminating this factor from Dr. Kjer-Petersen's standard deviation of 0.19 , we are left with a mean error, for Dr. Kjer-Petersen'e observations, of between 0.17 and $0 \cdot 18$, which we must attribute wholly to imperfections in his technique.

Having said this, we must examine the method by which our figures have been obtained. Most observers either knew, or were liable to know, when they were counting their "normals." This introduces the question of bias. Then, 100 cells were counted in most cases, but a second lot of 100 has been counted, at the observer's discretion, if the normals were found to disagree widely, or if the phagocytosis in any film appeared to be irregular. This introduces the question of reduction of error by taking larger samples. That neither this practice of revision, nor personal bias, has tended to reduce divergencies to any serious degree is the opinion of most practised observers. This opinion is supported by the following facts. (a) The close agreement of the distribution of our figures with the law of error. We lay stress on this, because Dr. Kjer-Petersen himself insists that "the influence of any predominant cause "will prevent a correspondence to the law of error. (b) During the last two months one of our fellow-workers, to avoid entirely the question of bias, has had his slides re-numbered and mixed up, being thas quite in the dark as to which of his slides were controls. During this time he has made 22 pairs of observations on normal sera, counting only 100 cells of each slide. The standard 
deviation for this series is $6 \cdot 6$ per eent, as opposed to 7 per cent. for the whole mass of our statistics. Further, it has occurred again and again that a result has been doubted by the observer on the score of bias, and has only been reaffirmed by a renewed count where this supposed source of error has been excluded.

3. The variations of tuberoulous sera are large.-We have given our reasons for believing that normal serum varies much less than Dr. Kjer-Petersen's figures might indicate. We now proceed to the consideration of the sera of tuberculous patients. We have been able to collect 931 observations on 319 patients who had not been inoculated. The standard deviation from the normal mean is 0.252 , a value greatly exceeding that found for normal sera $(0.07)$. The observations are distributed as follows, and agree very nearly with the "normal curve of error."

\section{TABLE II}

$$
\begin{aligned}
& \text { Within } 0.126 \text { of the normal mean } 374=402 \text { per mille. }
\end{aligned}
$$

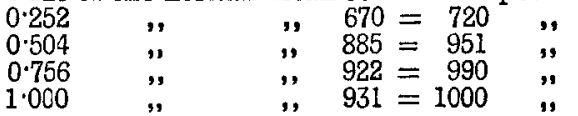

The opsonic indices of tuberculous patients vary, therefore, three or four times as widely as the indices of normal sera. Thus much we can conclude at present; but with increased accuracy of technique it may in the future be shown that the variations of normal sera are much less than we have here assumed, while the correction introduced by such improvements would have little effect on the larger value we have found for the variations of tuberculous sera.

Let us see to what corrections this figure is liable. In a minority of these determinations only 50 cells were counted, but this can only have altered the results very little. The mean counting error cannot be greater than the standard deviation of our observations of normal sera. This was 0.07 , 100 cells being counted. For counts of 50 cells the mean error will consequently not exceed $0 \cdot 10$. Applying this maximum correction to the whole number of observations, and also allowing for the mean error of the estimation of unity, the mean variation of pathological sera is only reduced from 0.252 to 0.226 . Such an alteration is trivial and without effect on the validity of our argument.

4. Some general considerations.-If statistical methods are to be applied we have shown that numbers are not wanting to support the truth of our conclusions. Further, Dr. KjerPetersen's experimental methods are open, even at this distance, to serious criticism. First, we suspect that a certain number of infected individuals may have been included among his 48 normals. In this laboratory, among those who have from time to time offered their blood as a standard in opsonic estimations, tubercle has been diagnosed seven times. The diagnosis rested in the first instance on variations of the tuberculo-opsonic index. In four of these cases clinically recognisable tuberculosis subsequently declared itself.

Dr. Kjer-Petersen also delegates the counting. of his slides to a second observer, and in so doing throws away a valuable check on the previous stages of the technique; a high level of perfection in making and staining films is only to be maintained by that constant self-criticism which is incidental to the actual counting of films one has oneself prepared.

\section{CHRONIC POLYCYTHÆMIA.}

By EDMUND CAUTLEY, M.D. Cantab., F.R.C.P. Lond., PHYSICTAN TO THE METROPOLITAN HOSPITAL AND THE BEIGRAVE HOSPITAL FOR CHILDREN.

THE following case of polycythæmia is worthy of record although the details do not add anything definite to our knowledge of the condition. The patient was under observation for a long time and a post-mortem examination was obtained. Vaquez first described a case in 1892 and ascribed it to congenital heart disease, until a necropsy in 1895 enabled him to exclude such a cause. Dr. J. W. Russell ${ }^{1}$ claims that he described the first case ${ }^{2}$ in England under the title of "An Unexplained Condition of Chronic Cyanosis." The patient was under the care of Dr. Robert Saundby, who gives a further description of in a paper on the subject. ${ }^{3}$

I The LaNoET, Dec. 8th, 1906, p. 1623.

2 THE LANCET, Feb. 22nd, 1902, p. 515.
3 Brit. Med. Jour., 1907, vol. i., 1165.
Dr. Saundby gives a full bibliography of recorded cases and mentions that Dr. F. Parkes Weber first named the affection "chronic splenomegalic polycythæmia." Since then a case in a man, aged 67 years, has been recorded by Pethybridge, ${ }^{4}$ and in a woman, aged 68 years, by McQuitty. ${ }^{5}$

The patient, a man, aged 47 years, was admitted into the Metropolitan Hospital under my care on Sept. 30th and was discharged on Oct. 25th, 1905. According to his past history he had an attack of hæmoptysis in 1887, influenza in 1893 and pnenmonia in 1901. He was an abstainer and denied lues venerea. For two months he had suffered from pain in the left side, difficulty in breathing, and constipation. He had had no congh. On examination he was found to be a healthy-looking man, with fresh complexion. His teeth were few and bad. The respirations were 34 and the pulse was 88 per minute. He had a subnormal temperature. The reflexes were normal. The heart dulness was diminished, the apex beat was normally placed, and the impulse was feeble. The liver dulness began at the lower border of the sixth rib. The left side of the chest was dall, below the level of the seventh rib in the nipple line and the eighth rib in the mid-axillary line, and the air entry was diminished. A tender lump was felt in the splenic region. It was shaped like the spleen and extended to within an inch of the middle line of the abdomen. It was continuous in dulness with the normal splenic dulness but was resonant over the lower part There was much tenderness on palpation and the muscles were rather rigid. A diagnosis of perisplenitis and pleurisy with slight effusion was made. A blood examination made on Oct. 19th showed : red cells, 7,500,000; and white cells, 50,000 . A week later the red cells were about the same in number and the white ones were 45,000. A differential count showed that the white cells were nearly all polymorphonuclears. The course of the illness was as follows. The spleen became much smaller and the tenderness disappeared. The pleural effusion cleared up slowly. The temperature was usually from a half to one degree below normal, the pulse-rate was from 72 to 88 , the respirations were 24 , and the urine was normal. The loss of weight was half a pound, On Nov. 15th the spleen was still palpable and the white cells numbered 39,000 . He had been at work for about three weeks and came to the hospital on account of pain in the right side and slight hrmoptysis.

In 1906 the man was again an in-patient from April 8th to July 14th, being admitted for pains on and off in the legs, thighs, and shoulders. The floridness of the face was more noticeable and on questioning he stated that he had had it for about two years. The colour varied from deep redness to a cyanotic tint. The ocular conjunctiva was injected and the palpebral intensely red. The tongue was clean, dry, and very red, and he complained of thirst. The cardiac dulness was diminished, the apex beat was not felt, and the sounds were clear. The spleen could be felt just beneath the costal margin and was slightly tender. Posteriorly its area of dulness was increased. The liver was rather enlarged and terder. The pulse-rate was 80 , the arteries were thickened and the arterial tension was high. The blood showed red cells, 9,800,000; hæmoglobin, 110 per cent.; white cells 82,000 , chiefly polymorphs. The fingers were a little clubbed. His symptoms were limited to weakness, variable pains and shortness of breath, and coldness of the hands and feet. On April 19th there was no pain in the limbs. Some hepatio pain and tenderness were present. On the 23rd the blood showed : red cells, 10,000,000; hæmoglobin, 130 ; and white cells, 59,000; the specific gravity was from 1068 to 1070 . The blood pressure was from 180 to 200 millimetres of mercury. The capillary reflex was 10 . On May 6th clubbing of the fingers was more marked. The patient had complained of tenderness over the liver and spleen, occipital and frontal headache, and tender gums with slight oozing of blood. He was more cyanosed. The blood pressure was 160 millimetres of mercury. There was much albumin in the urine. On the next day he had pain in the right vastus internus. The knee-jerks were anobtainable and the plantar reflex was flexor in character. On May 10th the tongue was moist and had a whitish fur on it. The spleen was not palpable. The urine was acid, of specific gravity 1015 , and contained much albumin. On the 17th the amount of albumin was reduced to a clond and the specific gravity was 1013. The blood showed: red cells, 8,600,000; hæmoglobin, 150 ; and some normoblasts. On the 24 th an attack of orchitis on the left side began and took nearly 